\title{
ON HEREDITARY INTERVAL ALGEBRAS
}

\author{
M. ALAMI and D. ZHANI
}

Received 19 June 2003

\begin{abstract}
We show that each hereditary interval algebra has a countable density and not conversely. Moreover, we show that, for an interval algebra, having countable density and being subalgebra of the interval algebra over the real line are equivalent statements.
\end{abstract}

2000 Mathematics Subject Classification: 06E05, 06E99, 54F05.

1. Introduction. Boolean algebras that are generated by subchains, that is, subsets that are linearly ordered under the Boolean partial order, were introduced in 1939 by Mostowski and Tarski [7] and have been extensively studied since then. Nowadays they are called interval algebras. All basic facts about these algebras can be found in [6, Section 15]. We remark, at this stage, that a subalgebra of an interval algebra need not be an interval algebra. For instance, one can consider the algebra of finite and co-finite subsets of the first uncountable cardinal. This leads us to the study of hereditary interval algebras, that is, those algebras of which any subalgebra is an interval algebra. The main concern of this note is to shed more light on these algebras. This note is organized as follows. Section 2 deals with definitions. Section 3 is a presentation of the main theorem. In Section 4, some consequences of this theorem are given.

2. Preliminaries. A partial ordered set $(T, \leq)$ is called a tree (resp., a pseudotree) whenever, for each element $t$ in $T,\{u \in T: u \leq t\}$ is a well-founded chain (resp., a chain). Throughout this note, we denote by $B(T)$ the tree algebra (resp., the pseudotree algebra on $T$ ), that is, the subalgebra of the power set $\mathscr{P}(T)$ generated by $\left\{b_{t}: t \in T\right\}$, where $b_{t} \stackrel{\text { def }}{=}\{u \in T: t \leq u\}$.

Theorem 16.7 in [6] enables us to look at trees with a single root without losing generality. Furthermore, if $T^{\prime}$ is a subtree of $T$, then $B\left(T^{\prime}\right)$ embeds into $B(T)$ by [6, Theorem 16.9]. These two facts remain valid for pseudotree algebras; for indications about the modifications of the arguments in [6] and for more on pseudotree algebras, we refer the reader to $[2,4]$. If $T$ is a chain, say $L$, then we usually denote $B(T)$ by $\operatorname{Int}(L)$ and we call $\operatorname{Int}(L)$ the interval algebra over $L$.

Now, let $C$ be a given chain and $a<b$ in $C$. We say that $b$ covers $a$ in $C$ whenever $[a, b[=\{a\}$. We then set $\operatorname{Gap}(C) \stackrel{\text { def }}{=}\{x: \exists a, b \in C[b$ covers $a$ and $x \in\{a, b\}]\}$. Next, recall the interval topology $\tau_{0}$ on $C$ generated by the set of open intervals $(a, b), a, b \in$ $C^{+}=C+\{\infty\}$; see [4]. Also, we say that a subset $X$ of $C$ is ordinarily dense in $C$ whenever $X$ is topologically dense in $\left(C, \tau_{0}\right)$ and $\operatorname{Gap}(C) \subseteq X$. 
Next, consider the following cardinal invariant on a Boolean algebra $B: \pi(B)=$ the algebraic density of $B=$ the minimal size of a dense subset of $B, d B=$ the topological density of $B=$ the minimal size of a dense subset of the Stone space of $B$, Ult $(B)$ (= the set of ultrafilters of $B$ endowed with Tychonoff's topology).

Also, for any topological space $X$, put $d X=\operatorname{Min}\{|D|: D$ dense in $X\}$. For more details, we refer the reader to [5]. Finally, recall that a separable topological space is a space that has a countable dense topological subspace, and for any subset $A$ of a topological space $X$, we denote by $\operatorname{cl}(A)$ the topological closure of $A$ in $X$; see [4].

3. Main result. We start with a definition.

DEFINITION 3.1. An infinite Boolean algebra is a hereditary interval algebra (h.i.algebra) whenever any subalgebra of it is an interval algebra.

Next, we state the main theorem of this note.

THEOREM 3.2. Let $B$ be an interval algebra and consider the following statements:

(i) $B$ is an h.i.-algebra,

(ii) $\pi(B)=\kappa_{0}$ and $B$ is an interval algebra,

(iii) $B=\operatorname{Int}(L)$, where $L$ is an infinite subset of the real line.

Then (ii) and (iii) are equivalent and (i) implies (ii).

The proof of this theorem will be a consequence of the following lemmas and propositions.

\section{Proof OF (i) IMPLIES (ii)}

Proposition 3.3. If $A$ is an h.i.-algebra, then neither $\omega_{1}$ nor $\omega_{1}^{*}$ embeds in $A$.

Proof. Assume the contrary. Without loss of generality, we may assume $\omega_{1} \leq A \stackrel{\text { def }}{=}$ $\operatorname{Int}(L)$ (since $A$ is an h.i.-algebra). Let $\left\langle a_{\alpha}: \alpha<\omega_{1}\right\rangle$ be an increasing continuous enumeration of $\omega_{1}$ and put $T=\left\{a_{\alpha}: \alpha<\omega_{1}\right\}$.

Next, define $b_{\alpha}=a_{\alpha+1} \bullet-a_{\alpha}$ for $\alpha<\omega_{1}$ and put $T_{0} \stackrel{\text { def }}{=}\left\{b_{\alpha}: \alpha<\omega_{1}\right\}(\subseteq A)$. Hence $B_{0}=\left\langle T_{0}\right\rangle$ is an uncountable subalgebra of $A$ which is isomorphic to an interval algebra since $A$ is an h.i.-algebra, but this is a contradiction since all chains in $B_{0}$ are countable.

Proposition 3.4. Let $B(T)$ be a tree algebra not embedding $\omega_{1}$ or $\omega_{1}^{*}$. Then every chain in $B(T)$ is at most countable.

Proof. Assume the proposition does not hold and let $C$ be a chain in $B(T)$ of size $\kappa_{1}$. Then, by [6, Theorem 16.20], $\omega_{1}$ or $\omega_{1}^{*}$ embeds in $B(T)$, which leads to a contradiction.

Proposition 3.5 (S. Koppelberg, J. D. Monk). Every interval algebra has a dense tree algebra.

Proof. Let $B=\operatorname{Int}(L)$. Hence $B$ is minimally generated by [3] and, by [3, Theorem 4.3] and the remark after [4, Corollary 2.4], $B$ has a dense subalgebra which is isomorphic to a tree algebra. 
Next, to end up the proof of (i) implies (ii), let $B$ be an h.i.-algebra. By Proposition 3.5, $B$ has a dense tree algebra, say $B(T)$. So it would be sufficient to show that $B(T)$ is countable. Indeed, by Proposition 3.3, $\omega_{1}$ and $\omega_{1}^{*}$ do not embed in $B$. Hence $\omega_{1}, \omega_{1}^{*}$ do not embed in $B(T)$. Now, by Proposition 3.4, every chain in $B(T)$ is countable. Again, since $B$ is an h.i.-algebra, $B(T)$ is in fact an interval algebra in which every chain is countable; that means $B(T)$ is countable. So, $\pi(B)=\kappa_{0}$. This completes the proof of (i) implies (ii).

Proof of (iii) IMPLIES (ii). Let $L$ be isomorphic to a subchain $C$ of $\mathbb{R}$. Hence $B=$ $\operatorname{Int}(L) \cong \operatorname{Int}(C) \leq \operatorname{Int}(\mathbb{R})$. But $\pi(\operatorname{Int}(C))=\aleph_{0}$ by [1, Proposition 1.5]. So, $\pi(B)=\aleph_{0}$.

Proof OF (ii) IMPLIES (iii). Next, we state the well-known result about the set of real line. See, for example, [12, Corollary 3.2].

LEMMA 3.6. The following statements are equivalent for any infinite set A:

(i) $A$ is a subchain of $\mathbb{R}$,

(ii) there is $B \subseteq A$ countable and ordinarily dense in $A$.

Let $B$ be an interval algebra such that $\pi(B)=\aleph_{0}, B=\operatorname{Int}(L)$ for some chain $L$. Now pick $B_{0}$ a countable dense subalgebra of $B$, and for any $a \in B_{0}$, written under its normal form,

$$
a=\bigcup_{i=1}^{n}\left[x_{i}, y_{i}\left[, \quad x_{i}, y_{i} \in L^{+}=L+\{\infty\} .\right.\right.
$$

We set $\operatorname{rel}(a) \stackrel{\text { def }}{=}\left\{x_{i}, y_{i}: i=1, \ldots, n\right\}$. Now, if $b$ covers $a$ in $L$, by denseness of $B_{0}$ in $\operatorname{Int}(L)$, there is a nonzero element $x_{0} \in B_{0}$ such that $x_{0} \subseteq\left[a, b\left[=\{a\}\right.\right.$. Hence $x_{0}=$ $\left[a, b\left[\in B_{0}\right.\right.$. So the set $L_{0}=\left\{\operatorname{rel}(x): x \in B_{0}\right\}$ is at most countable (since $\left|B_{0}\right|=\aleph_{0}$ ) and $\operatorname{Gap}(L) \subseteq L_{0}$.

Now, by the above lemma, it is sufficient to show that $L_{0}$ is topologically dense in $L$. To this end, let $x_{0} \in L$ and let $(a, b)$ be an open interval in $L$ containing $x_{0}$.

We need to show that $(a, b) \cap L_{0} \neq \varnothing$.

CASE 1. $x_{0}$ covers $a$ or $b$ covers $x_{0}$.

In this case, $x_{0} \in L_{0}$, and hence $(a, b) \cap L_{0} \neq \varnothing$. This takes care of Case 1 .

CASE 2 (Case 1 fails). There are $a^{\prime}, b^{\prime} \in L$ such that $a<a^{\prime}<x_{0}<b^{\prime}<b$. Thus $\left[a^{\prime}, b^{\prime}[\in\right.$ $\operatorname{Int}(L)$, and then, by denseness of $B_{0} \operatorname{in} \operatorname{Int}(L)$, pick a nonzero element $y_{0} \in B_{0}$ such that $y_{0} \subseteq\left[a^{\prime}, b^{\prime}\right.$. So $\left[a^{\prime}, b^{\prime}\left[\cap L_{0} \neq \varnothing\right.\right.$. Thus $(a, b) \cap L_{0} \neq \varnothing$. This takes care of Case 2 .

Hence the proof of (ii) implies (iii) is finished.

4. Consequences. In this section, some corollaries of the main theorem are given.

COROLLARY 4.1. If $B(T)$ is an uncountable h.i-algebra, then $\eta \leq B(T)$, where $\eta$ is the order type of the set of rationals under their natural ordering.

Proof. $B(T)$ is an h.i.-algebra, say $\operatorname{Int}(L)$. Hence, by Proposition $2.3, \omega_{1}$ and $\omega_{1}^{*}$ do not embed in $L$. Hence, by [11, Corollary 5.30], $\eta \leq L$, and therefore $\eta \leq B(T)$. 
COROLLARY 4.2. If $B$ is an interval algebra of uncountable density, that is, $\pi(B) \neq \mathrm{N}_{0}$, then there is a subalgebra $B_{0}$ of $B$ which is not an interval algebra.

COROLLARY 4.3. There is an interval algebra satisfying the condition in Corollary 4.2 .

To see this, take, for example, $\operatorname{Int}(L)$ with $L=\omega_{1}$. Notice that $\operatorname{Int}\left(\omega_{1}\right)$ is a superatomic algebra. For an atomless interval algebra, one can take $\operatorname{Int}(L)$ with $L=\eta$. $\omega_{1}$.

REMARK 4.4. First notice that in the main theorem, we showed that (i) implies (ii) and by a result of Nikiel (see, e.g., [8, Theorem 3.1]), (i) and (ii) are not equivalent statements in the main theorem. For instance, an erratum appeared in [9] showing that the proof of [8, Theorem 3.1] is wrong. To see this, we consider the following counterexample that appeared in [10] and that was communicated to us by Professor L. Heindorf.

Let $\mathbb{Q}$ denote the subset of all rational numbers and $\mathbb{P}$ the subset of all irrational numbers of $] 0,1[$. Let $Y=([0,1] \times\{0\}) \cup(\mathbb{P} \times\{1\})$, let $\leq$ denote the lexicographic ordering on $Y$, and take $Y$ with its order topology. Then $Y$ is a linearly ordered compact space and $\mathbb{Q} \times\{0\}$ is a dense subset.

Let $X=Y \cup \mathbb{Q}$ with the following topology: the points of $\mathbb{Q}$ are isolated and basic neighborhoods of each $(t, i) \in Y$ are of the form $U \cup\{s \in \mathbb{Q}: s \neq t$ and $(s, i) \in U\}$, where $U$ is an open neighborhood of $(t, i)$ in $Y$. One can see that $X$ is a boolean separable space, a continuous image of an orderable space, and yet not orderable.

ACKNOWLEDGMENTS. The authors would like to thank the referee and also Professor L. Heindorf for their helpful comments and suggestions.

\section{REFERENCES}

[1] M. Bekkali, Chains and antichains in interval algebras, J. Symbolic Logic 59 (1994), no. 3, 860-867.

[2] _ Pseudo treealgebras, Notre Dame J. Formal Logic 42 (2001), no. 2, 101-108.

[3] S. Koppelberg, Minimally generated Boolean algebras, Order 5 (1989), no. 4, 393-406.

[4] S. Koppelberg and J. D. Monk, Pseudo-trees and Boolean algebras, Order 8 (1991/1992), no. $4,359-374$.

[5] J. D. Monk, Cardinal Functions on Boolean Algebras, Lectures in Mathematics ETH Zürich, Birkhäuser Verlag, Basel, 1990.

[6] J. D. Monk and R. Bonnet (eds.), Handbook of Boolean Algebras. Vol. 3, North-Holland Publishing, Amsterdam, 1989.

[7] A. Mostowski and A. Tarski, Boolesche ringe mit geordneter basis, Fund. Math. 32 (1939), 69-86 (German).

[8] J. Nikiel, Orderability properties of a zero-dimensional space which is a continuous image of an ordered compactum, Topology Appl. 31 (1989), no. 3, 269-276.

[9] _ Erratum: "Orderability properties of a zero-dimensional space which is a continuous image of an ordered compactum", Topology Appl. 36 (1990), no. 1, 93.

[10] J. Nikiel, S. Purisch, and L. B. Treybig, Separable zero-dimensional spaces which are continuous images of ordered compacta, Houston J. Math. 24 (1998), no. 1, 45-56.

[11] J. G. Rosenstein, Linear Orderings, Pure and Applied Mathematics, vol. 98, Academic Press, New York, 1982 . 
[12] S. Todorčević, Trees and linearly ordered sets, Handbook of Set-Theoretic Topology, NorthHolland Publishing, Amsterdam, 1984, pp. 235-293.

M. Alami: Department of Mathematics, Faculty of Sciences and Technology, Route D'Immouzer, B. P. 2202, Fez, Morocco

E-mail address: alami@fstf.ma

D. Zhani: Department of Mathematics, Faculty of Sciences Dhar El Mehrez, B. P. 1796, Fez, Morocco

E-mail address: dzhani@fsdmfes.ac.ma 


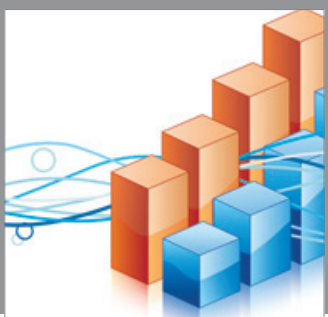

Advances in

Operations Research

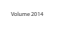

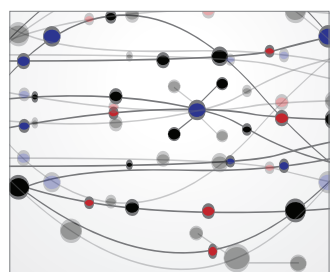

\section{The Scientific} World Journal
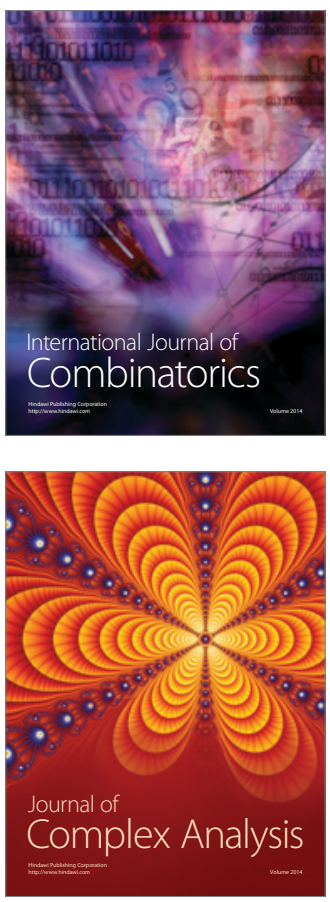

International Journal of

Mathematics and

Mathematical

Sciences
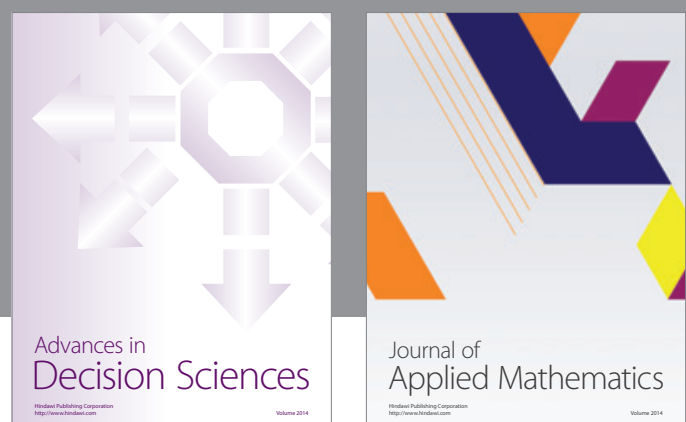

Journal of

Applied Mathematics
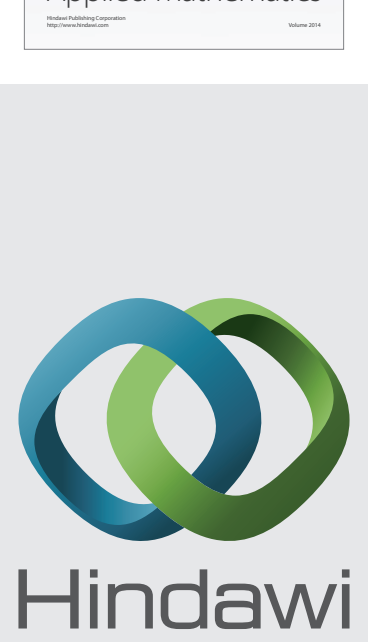

Submit your manuscripts at http://www.hindawi.com
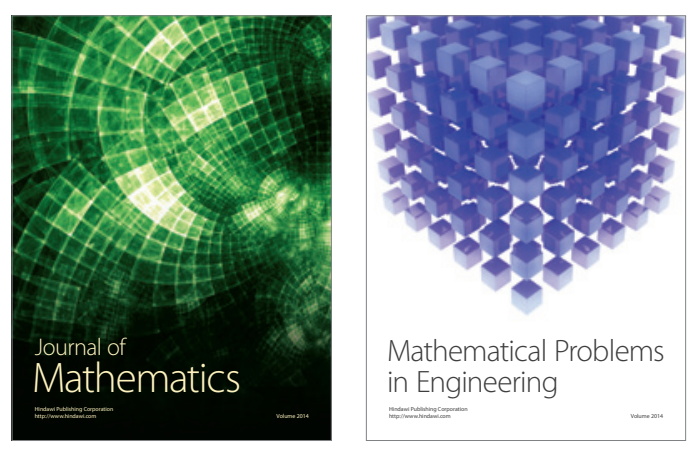

Mathematical Problems in Engineering
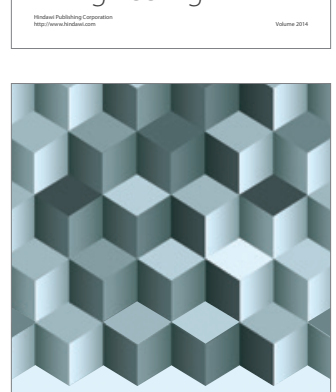

Journal of

Function Spaces
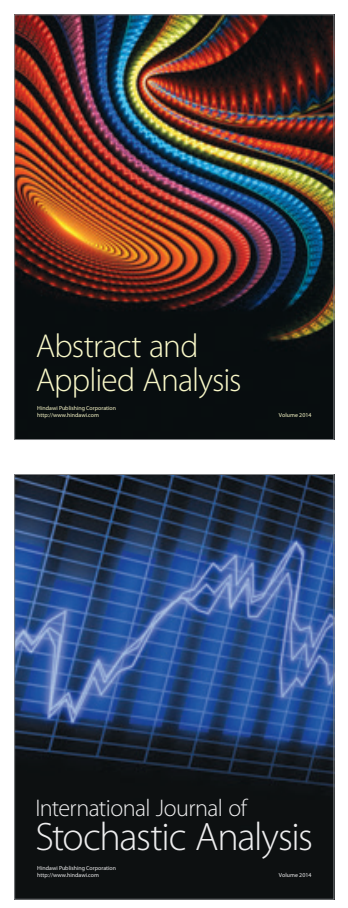

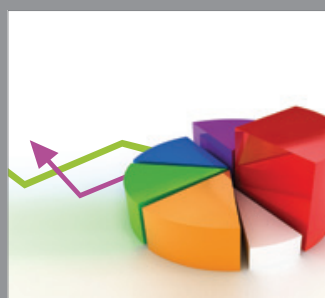

ournal of

Probability and Statistics

Promensencen
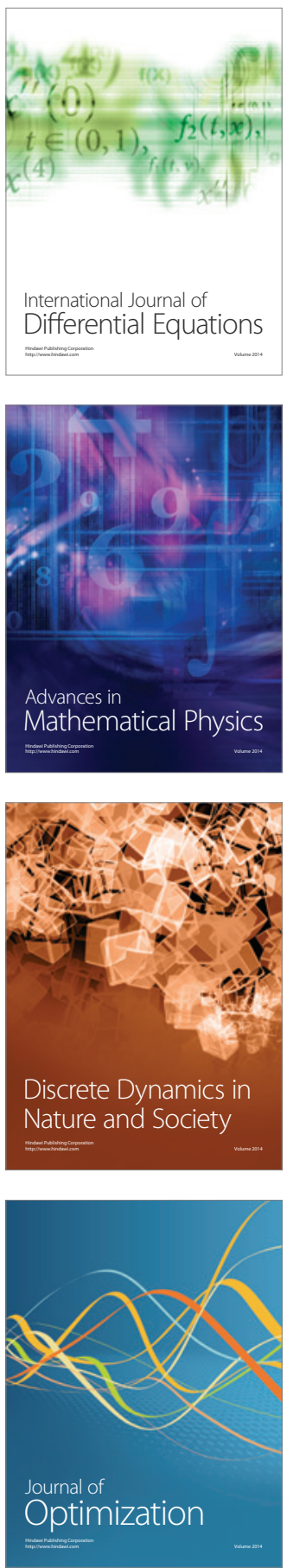